\title{
POPULATION STUDY ARTICLE Clinical and social factors associated with excess weight in Hispanic and non-Hispanic White children
}

\author{
Sahel Hazrati ${ }^{1}$, Farah Khan ${ }^{2}$, Kathi Huddleston ${ }^{1}$, Faith De La Cruz ${ }^{1}$, John F. Deeken ${ }^{1}$, Alma Fuller ${ }^{1}$, Wendy S. W. Wong ${ }^{1}$, \\ John E. Niederhuber ${ }^{1}$ and Suchitra K. Hourigan ${ }^{1}$
}

BACKGROUND: Hispanic children are disproportionately affected by obesity, with this disparity starting at a young age, and there is a paucity of data comparing factors associated with excess weight in the first year of life in Hispanic vs. non-Hispanic populations. METHODS: Excess weight was defined as weight-for-length $\geq 95$ th percentile. The associations of potential risk factors were compared by ethnicity stratification.

RESULTS: Of the 1009 children, 302 (30.0\%) were Hispanic and 707 (70.0\%) were non-Hispanic White. The rate of excess weight was $30.1 \%$ and $13.6 \%$ among Hispanic and non-Hispanic White children, respectively. Factors associated with excess weight for nonHispanic White children were higher than recommended weight gain during pregnancy (odds ratio (OR) 1.8 (1.2-3.1)), higher paternal body mass index (BMI) (OR 1.1 (1.02-1.15)), higher birth weight (OR 1.001 (1.001-1.002)), and lower breast milk feedings at 6 months (OR $0.98(0.96-0.98)$ ). Factors associated with excess weight for Hispanic children were lower maternal education (OR 2.37 (1.1-4.5)) and lower breast milk feedings at 6 months (OR 0.98 (0.96-0.99)).

CONCLUSION: There are differential risk factors associated with excess weight at 12 months between Hispanic and non-Hispanic White children. Identification of differential factors in different ethnicities may allow for more targeted anticipatory guidance reduce obesity in at-risk populations.

Pediatric Research (2019) 85:256-261; https://doi.org/10.1038/s41390-018-0264-9

\section{INTRODUCTION}

Childhood obesity has become a critical public health concern both in the United States and worldwide. Over the past three decades, the prevalence of obesity among United States youth has nearly tripled to $17 \%$, with an overall prevalence of $8.9 \%$ among preschool-aged children., ${ }^{1,2}$ Hispanic children are disproportionately affected by obesity, with this disparity starting at a young age; in children aged 2-5 years the prevalence of obesity between 2011 and 2014 was $15.6 \%$ in the Hispanic population vs. $5.2 \%$ in the non-Hispanic White population. ${ }^{1}$

Childhood obesity is multifactorial, and clinical, social, and genomic factors contribute to this disease $\mathrm{e}^{3-5}$. It has long been established that childhood obesity is also associated with obesity later in life. ${ }^{6-8}$ In the US National Longitudinal Study of Adolescent Health, nearly $40 \%$ of obese adolescents (with a body mass index (BMI) $>95$ th percentile) became severely obese (BMI $>40 \mathrm{~kg} / \mathrm{m}^{2}$ ) by 30 years of age compared with $<5 \%$ of normal weight teenagers. ${ }^{7,9}$ Very early infant and childhood obesity also predicts obesity in later childhood ${ }^{10-12}$ and adulthood, ${ }^{12,13}$ with a critical window in very early life hypothesized to be especially predictive of later obesity risk ${ }^{14}$ due to the metabolic programming and epigenetic changes that can occur during early neonatal growth and development. ${ }^{15}$

Obesity rates for Hispanic children are higher starting at a younger age. ${ }^{1}$ Previous studies have shown that rapid weight gain in infancy has been associated with childhood obesity ${ }^{14}$ and is often one of the strongest predictors of obesity later in childhood.
Despite such observations, there is a paucity of data comparing factors associated with being overweight or obese in the first year of life in Hispanic vs. non-Hispanic populations.

Inova's "The First 1000 Days of Life and Beyond" cohort study, consisting of $>3500$ families, provides an opportunity for analysis and overview of key issues related to pediatric health conditions including childhood obesity and health disparities. In this manuscript, we report the results of a cross-sectional analysis within this cohort with the aim to investigate whether there are differential risk factors for obesity among 12-month-old Hispanic vs. nonHispanic White children and to evaluate the genetic ancestry background of both cohorts.

\section{METHODS}

Study design

"The First 1000 Days of Life and Beyond" study is a childhood longitudinal cohort based in the Inova Health System. Beginning in July 2012, women have been recruited during pregnancy to participate in this study. Inclusion criteria included maternal age $>18$ years, a willingness to participate in longitudinal surveys every 6 months and to have whole-genome sequencing of the family trio-mother, father, and baby. Institutional Review Board (IRB) approval was obtained (WIRB\#20120204, Inova IRB\#15-1804). ${ }^{16}$ This study is based on 12-month surveys completed by 1915 study participants. The 355 children who had missing anthropometrics were excluded. Outliers were removed for reported lengths using

${ }^{1}$ Inova Translational Medicine Institute, Falls Church, VA, USA and ${ }^{2}$ Inova Children's Hospital, Falls Church, VA, USA

Correspondence: Sahel Hazrati (Sahel.hazrati@inova.org)

These authors contributed equally: Sahel Hazrati, Farah Khan

Received: 14 August 2018 Revised: 1 November 2018 Accepted: 5 November 2018

Published online: 10 January 2019 
the interquartile range rule as previously validated, ${ }^{17}$ and five subjects were excluded due to medical conditions associated with disordered growth patterns (Trisomy 21, hypothyroidism and Ogden syndrome). Self-reported maternal ethnicity was recorded; only those with self-reported Hispanic or non-Hispanic White ethnicity were included. The remaining 1009 children were included, of which 302 were Hispanic and 707 were nonHispanic White children.

Characteristics of mothers, fathers, and children

Clinical, social, and environmental data were collected using surveys, questionnaires, and medical records. Sociodemographic factors including maternal ethnicity and education were collected through questionnaires. Information about pregnancy complications such as gestational diabetes, children's birth weight, gestational age, parity, and sex were obtained from medical records. Nutritional data were collected using an adopted and modified version of the CDC-Behavioral Risk Factor Surveillance System; sleep and screen time data were collected through surveys at 6 and 12 months. Parentally reported anthropometrics were collected using surveys at 12 months of age. Maternal stress score was evaluated using Perceived Stress Scale at 12-month survey. ${ }^{18}$ The congruence of paternal ethnicity and maternal ethnicity was examined.

Of 1009 children, only 543 had genomic data. The ancestry of the 543 children including 194 (35.7\%) Hispanic and 349 (64.3\%) non-Hispanic White children were estimated by the ancestry and kinship toolkit $\mathrm{AKT}^{19}$ using 17,535 reliable and common singlenucleotide polymorphisms by projecting the samples into the 1000 genomes $^{20}$ principal components analysis, followed by using the known five super populations (European (EUR), African (AFR), Admixed American (AMR), South Asian (SAS), and East Asian (EAS)) to cluster and assign admixture proportions.

Gestational weight gain recommendations were categorized into three groups: "lower than recommended range," "in the recommended range," and "higher than recommended" range. Weight gain during pregnancy was calculated using prepregnancy and admission weight at delivery. The National Academies, Health, and Medicine Division recommendations for weight gain were used to determine if mothers met the recommended weight gain for their pre-pregnancy BMI. ${ }^{21}$ Adherence to American Academy of Pediatrics (AAP) guideline for solid food introduction was evaluated whether there was exclusive breast milk feeding or formula without solid food introduction until 6 months of age. Weight-for-length (WFL) percentiles at 12 months were calculated using the World Health Organization sex-specific growth charts and parentally reported anthropometrics. ${ }^{22}$ To be included in the study, the survey had to be returned within 2 months of when a child turned 12 months. A corrected age of 12 months was used for children who were born prematurely. Based on calculated WFL percentiles, children were classified as obese ( $\geq 95$ th percentile), or normal weight $(<95$ th percentile). Mean substitution technique was used for fathers' BMI. $^{23}$

\section{Statistical analysis}

Prevalence of WFL status of $<95$ th and $\geq 95$ th percentile groups was described and compared among Hispanic and not Hispanic White children using $x^{2}$ test. The characteristics of the study cohort and potential risk factors of obesity were compared by ethnicity using their mean, standard deviations, and frequencies for continuous and categorical variables, respectively. Association of WFL status of $<95$ th and $\geq 95$ th percentile to ethnicity was evaluated, due to high congruence between maternal and paternal ethnicity stratification by maternal ethnicity was used to produce two groups including non-Hispanic White and Hispanic children to control for the confounding effect of ethnicity on excess weight and to evaluate the exposure-outcome association within each ethnicity. ${ }^{24}$ Potential predictive prenatal, parental, and obesogenic risk factors of excess weight for Hispanic and non-Hispanic White children were examined separately using multiple logistic regression models. Forest plots were generated from pre-computed odds ratio and confidence intervals based on the logistic regression results. In order to evaluate the congruence of self-reported maternal ethnicity and children's ancestry admixture, genomic-derived ancestry admixture of children was used to create two clusters by using the $k$-means clustering technique. ${ }^{25}$ Congruence of self-reported maternal ethnicity and children's ancestry admixture generated clusters was evaluated using $X^{2}$ test. Distribution of admixture proportion was visualized for children of Hispanic and non-Hispanic White mothers. Furthermore, distribution of admixture proportion among normal and obese children was assessed. All statistical analyses were performed using R 3.1.2 (R Project for Statistical Computing, Vienna, Austria) and SAS 9.4 (SAS Institute Inc., Cary, NC, USA).

\section{RESULTS}

Of the 1009 children included in the study, 302 (30.0\%) were Hispanic and 707 (70.0\%) were non-Hispanic White. The rate of excess weight was $30.1 \%$ among Hispanic children as compared to $13.6 \%$ among non-Hispanic White children ( $p$ values $<0.0001)$ (Fig. 1)

Table 1 compares prenatal, parental, and children's characteristics along with obesogenic risk factors among all Hispanic and non-Hispanic White children. The Hispanic group compared to the non-Hispanic White Group had a lower maternal age, higher maternal BMI, lower cesarean section delivery rate, lower maternal stress, higher household count, differing compliance with American College of Obstetricians and Gynecologist guidelines for weight gain during pregnancy (more Hispanic population with lower than recommended weight gain, more in non-Hispanic White cohort of higher than recommended weight gain), higher parity, lower education, and lower paternal age ( $p$ value $<0.0001)$. The Hispanic group also had a higher gestational diabetes rate ( $p$ value $=0.001$ ). Dietary variables including frequency of juice consumption and French fries consumption were significantly higher among Hispanic children; however, breast milk frequency at 6 months was significantly lower among Hispanic children ( $p$ value $<0.0001$ ). More non-Hispanic White children had solid food introduction delayed until after 6 months ( $p$ value $<0.001$ ). Hispanic children were also reported to have higher weight gain in first year of life and to have fewer feeding difficulties ( $p$ value $=$ 0.006).

Results from multiple logistic regression models revealed that factors associated with excess weight for non-Hispanic White children were higher than recommended weight

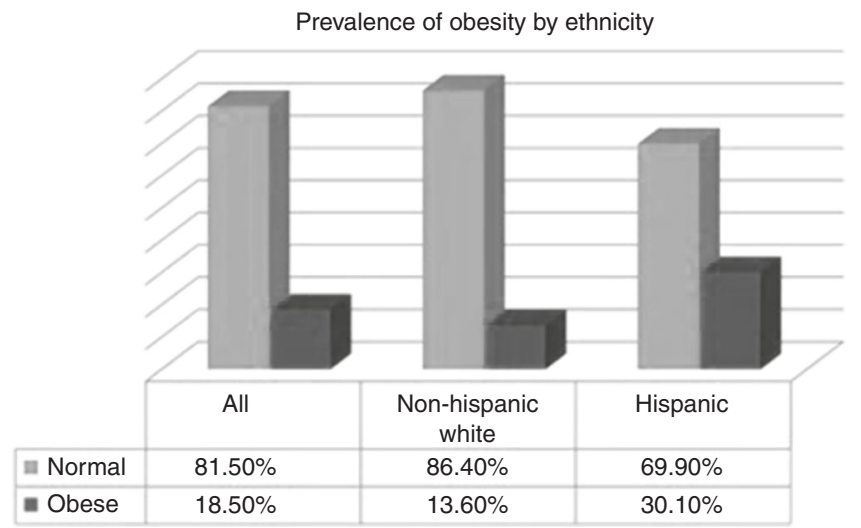

Fig. 1 Prevalence of obesity by ethnicity. Obesity was highest in the Hispanic population 
Table 1. Prenatal, parental, and children's characteristics along with obesogenic risk factors among all Hispanic and non-Hispanic White children

\begin{tabular}{|c|c|c|c|}
\hline Characteristics and obesogenic risk factors & $\begin{array}{l}\text { Non-Hispanic White }(N=707) \text {, } \\
\text { mean }(S D) \text { or } \%\end{array}$ & $\begin{array}{l}\text { Hispanic }(N=302) \text {, } \\
\text { mean }(S D) \text { or } \%\end{array}$ & $p$ Value \\
\hline Maternal age (years) & $32.8(3.9)$ & $30.2(5.3)$ & $<0.0001$ \\
\hline \multicolumn{4}{|l|}{ Delivery mode } \\
\hline Cesarean section: repeat & 25.7 & 20.2 & \multirow[t]{2}{*}{$<0.0001$} \\
\hline Cesarean section: primary & 25.5 & 11.3 & \\
\hline Preeclampsia/PIH/HELLP & 9.3 & 6.6 & 0.1 \\
\hline Gestational diabetes & 5.4 & 10.9 & 0.001 \\
\hline \multicolumn{4}{|l|}{ ACOG guideline for weight gain during pregnancy } \\
\hline Lower than recommended range & 11.0 & 23.7 & \multirow[t]{3}{*}{$<0.0001$} \\
\hline In the recommended range & 35.9 & 30.1 & \\
\hline Higher than recommended range (pre-term birth excluded) & 53.1 & 46.2 & \\
\hline $3^{+}$ & 15.5 & 35.4 & $<0.0001$ \\
\hline \multicolumn{4}{|l|}{ Maternal education } \\
\hline Less than associate degree & 3.8 & 66.2 & \multirow[t]{3}{*}{$<0.0001$} \\
\hline Associate degree or higher & 92.1 & 22.8 & \\
\hline Missing & 4.1 & 11.0 & \\
\hline Perceived stress scale & $12.0(5.7)$ & $9.1(5.8)$ & $<0.0001$ \\
\hline Household count & $4(1)$ & $5(2)$ & $<0.0001$ \\
\hline Paternal age (years) & $35.0(5.1)$ & $32.7(6.0)$ & $<0.0001$ \\
\hline Paternal body mass index $\left(\mathrm{kg} / \mathrm{m}^{2}\right)$ & $27.4(4.2)$ & $27.9(4.0)$ & 0.08 \\
\hline Birth weight $(g)$ & $3350.2(582.1)$ & $3323.2(497.7)$ & 0.4533 \\
\hline Breast milk feeds (frequency/week at $6 \mathrm{M}$ ) & $27.4(22.1)$ & $18.3(23.1)$ & $<0.0001$ \\
\hline Juice consumption (frequency/week at $12 \mathrm{M}$ ) & $0.4(1.1)$ & $1.7(2.0)$ & $<0.0001$ \\
\hline French fries (frequency/week at $12 \mathrm{M}$ ) & $0.6(0.9)$ & $0.3(0.6)$ & $<0.0001$ \\
\hline Feeding difficulties (yes) & 9.9 & 4.7 & 0.006 \\
\hline TV and video time (never) & 17.8 & 15.2 & 0.3 \\
\hline
\end{tabular}

ACOG American College of Obstetricians and Gynecologist, AAP American Academy of Pediatrics, HELLP hemolysis, elevated liver enzyme levels, and low platelet count, PIH pregnancy induced hypertension

gain during pregnancy (OR $1.8(1.2-3.1))$, higher paternal BMI (OR 1.1 (1.02-1.15)), higher birth weight (OR 1.001 (1.001-1.002)), and lower breast milk feedings at 6 months (OR 0.98 (0.96-0.98)) (Fig. 2a). On the other hand, factors associated with excess weight for Hispanic children were lower maternal education (OR 2.37 (1.1-4.5.)), and similar to nonHispanic White children, increased breast milk feedings at 6 months was a protective factor against being obese (OR 0.98 (0.96-0.99)) (Fig. 2b).

The association between maternal self-reported ethnicity and the genetic admixture of the child was examined. The Hispanic population is a mixture of mainly EUR and AMR, with some portion of AFR descent. Non-Hispanic Whites are mostly of EUR decent, although 11 individuals ( 3\%) had more than 10\% AMR proportions (Fig. 3).

In order to examine the congruence between self-reported maternal ethnicity and children's genetic ancestry clusters were generated. Two clusters were generated from genomic-derived ancestry admixture proportion from whole-genome sequencing of the 543 children with this information available. Cluster 1 means were EUR (0.327), AMR (0.551), AFR (0.075), EAS (0.028), and SAS (0.016) and cluster 2 means were EUR (0.901), AMR (0.024), AFR (0.021), EAS (0.013), and SAS (0.039). Cluster 1 included $82.5 \%$ of children of Hispanic mothers and cluster 2 included $99.7 \%$ of children of non-Hispanic White mothers. 
a

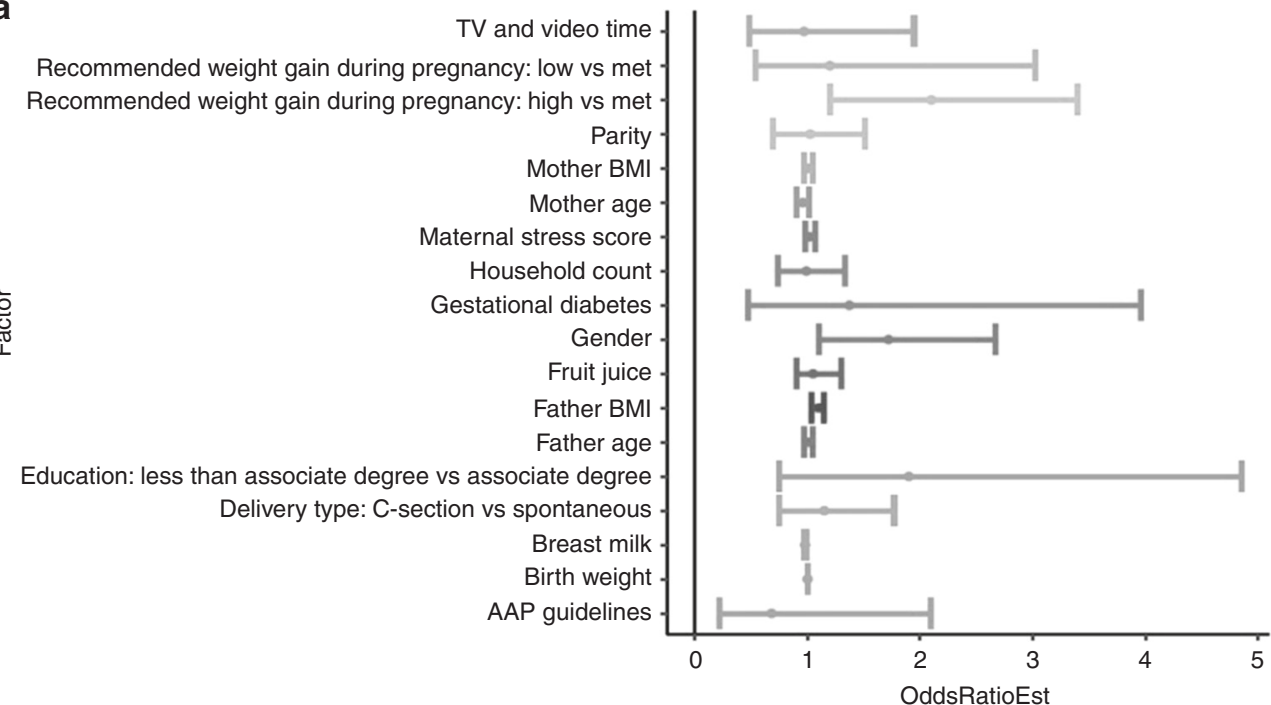

b

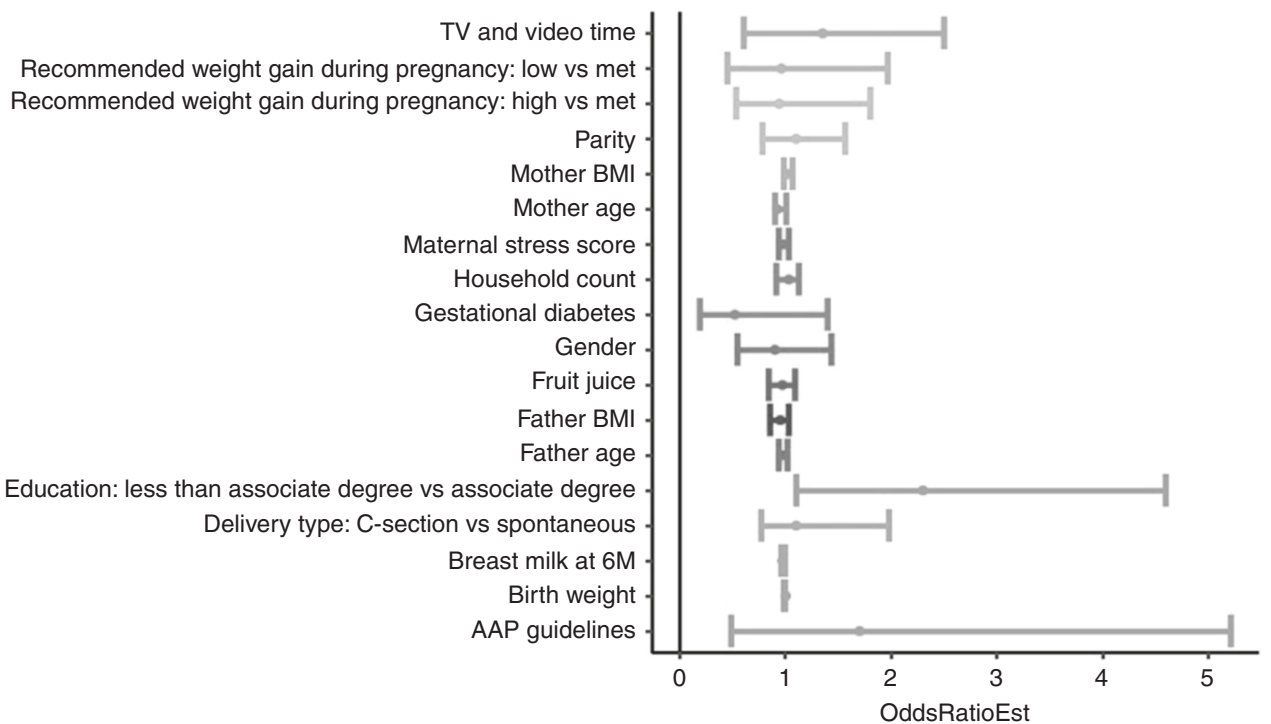

Fig. 2 a Forest plot showing factors associated with obesity for non-Hispanic White children. b Forest plot showing factors associated with obesity for Hispanic children

\section{DISCUSSION}

Obesity and excess weight is disproportionately higher in the Hispanic population, even in very early childhood. Different clinical and social factors are associated with excess weight at 12 months of age in Hispanic and non-Hispanic children.

The rate of excess weight in the Hispanic population was strikingly higher in this cohort, even at the very young age of 12 months, as previously reported in older children. ${ }^{2}$ Although obesogenic risk factors for this group of children as a whole were identified, as previously described, ${ }^{16}$ when stratified by ethnicity, differential risk factors for each cohort were found.

Surprisingly, within the Hispanic cohort there was no statistically significant association with higher than recommended weight gain during pregnancy as seen in the non-Hispanic cohort. Studies have shown that an excessive amount of weight gain during pregnancy is associated with an increased risk for obesity in childhood and adolescence. ${ }^{26}$ It has previously been noted that Hispanic women and non-Hispanic black women generally begin their pregnancy with a higher likelihood of being overweight or obese and have lower weight gain during pregnancy compared with non-Hispanic White women who were most likely to gain weight during pregnancy above the current recommendations. ${ }^{27}$ This was also seen in our study. This raises the question of whether there should be different recommendations for weight gain in pregnancy based on ethnicity, as more focus is brought towards the known prevalence of obesity across different ethnic groups. ${ }^{28}$ It could also be postulated that intrauterine fetal overnutrition has different hormonal, microbiome, immune modeling, and epigenetic influences in different ethnicities. ${ }^{29,30}$ It is also interesting that higher paternal BMI is associated with excess weight in nonHispanic White children, but not in Hispanic children. To our knowledge, this has not been reported before and the biological reason for this is unclear and warrants further exploration. It is known that there is stronger influence of maternal obesity than paternal obesity on infant body mass ${ }^{31}$ and that the Mother's genetic contribution to early childhood body mass may be higher than the Father's; ${ }^{32}$ it could be hypothesized that the paternal genetic contribution may also vary with ethnicity.

While higher birth weight is a recognized risk factor for very early childhood obesity, ${ }^{16}$ in our study it was only associated with obesity in the non-Hispanic White population, not the Hispanic population. It is possible that postnatal obesogenic exposures may 


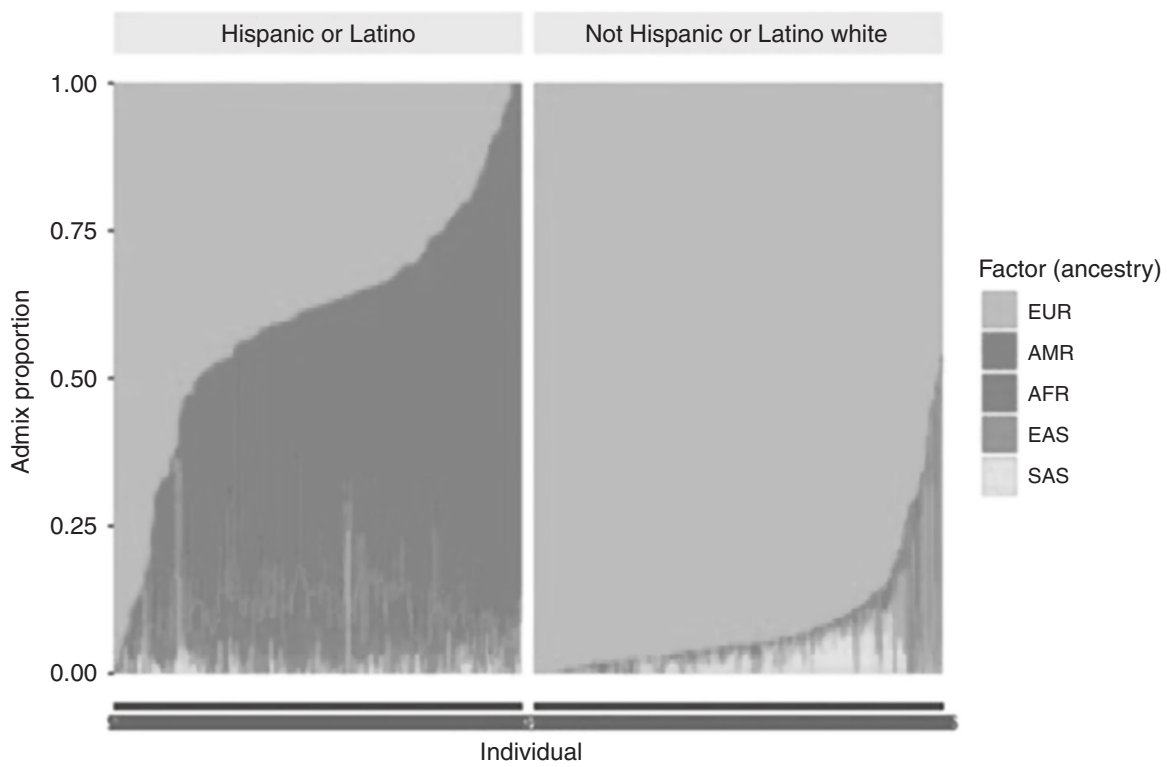

Fig. 3 Admixture proportion for Hispanic vs. non-Hispanic White children

play a larger role in the Hispanic population. Indeed, rapid infant weight gain has been reported as a risk factor for later childhood obesity in this Hispanic population. ${ }^{33}$

Often, presumptions are made on the part of health-care providers that early introduction by Hispanic mothers of sugary beverages is done to promote the development of infant taste and results in subsequent weight gain. ${ }^{34}$ While juice consumption was significantly higher in the Hispanic cohort, it was not associated with obesity after stratification in either cohort at this young age, possibly due to small overall amounts being consumed at this age. However, early juice introduction offers no health benefits, and current recommendations to limit juice consumption in early childhood in all populations should be reinforced. $^{35}$

Lower maternal education was associated with obesity in the Hispanic cohort only; however, this data point is difficult to draw conclusions from as $66.2 \%$ of the mothers of the Hispanic cohort had an education less than an Associate's degree compared to $3.2 \%$ of non-Hispanic White women in our study. Of note, previous studies have reported higher maternal education attainment as a potentially protective factor against childhood obesity. ${ }^{36}$

Receiving more breastmilk at 6 months of age was protective against obesity at 12 months of age in both cohorts. However, there was a significantly lower amount of breastmilk given in the Hispanic cohort compared with the non-Hispanic White cohort. Studies have shown that Hispanic mothers are more likely to practice non-exclusive breastfeeding and perceive chubbier infants as healthy infants. ${ }^{8,37-39}$ This is just one example of how different cultural norms drive feeding practices in Hispanic children, and how they may not benefit from breast milk's protective effect against obesity.

To our knowledge, this is the first study to evaluate the genetic admixture of the study subjects at 12 months. While the Hispanic population was mainly a mixture of EUR and AMR, with a small portion of AFR descent, the non-Hispanic Whites were mostly of EUR descent. It is well known that the obesity rate amongst the Hispanic population is higher-this was re-demonstrated in our study-however, this highlights an underlying genetic predisposition towards being obese in addition to social and cultural factors.

Strengths of our study included having prospectively collected data on a wide variety of risk factors extending from parental to prenatal to early childhood, and the availability of genetic admixture data. However, this study also has several potential limitations. All 12-month height and weight data were based on parental self-report. However, this has been validated in this study cohort. ${ }^{17}$ Furthermore, self-reported maternal ethnicity, which is widely used in childhood obesity studies, ${ }^{16,40,41}$ was also used in our study rather than the child's ethnicity. In an attempt to reconcile this difference, genetic admixture from the child was used and there was a high correlation between this and maternal self-reported ethnicity. Unfortunately, genomic data was not available for all of the children in the study.

\section{CONCLUSION/FUTURE DIRECTION}

Our study shows that there are differential risk factors associated with excess weight at 12 months between Hispanic and nonHispanic White children. Identification of differential risk factors in different ethnicities may allow for more targeted and personalized anticipatory guidance to try and reduce obesity in at-risk populations. Whether these differential risk factors for obesity remain later in life will be followed in this longitudinal study.

\section{ACKNOWLEDGMENTS}

Thank you to the Richard H. Schwartz Pediatric Resident Research and Education for support of this work.

\section{ADDITIONAL INFORMATION}

Competing interests: The authors declare no competing interests.

Publisher's note: Springer Nature remains neutral with regard to jurisdictional claims in published maps and institutional affiliations.

\section{REFERENCES}

1. Ogden, C. L. et al. Trends in obesity prevalence among children and adolescents in the United States 1988-994 through 2013-2014. JAMA. 315, 2292-2299 (2016).

2. Ogden, C. L. et al. Prevalence of Obesity Among Adults and Youth: United States, 2011-2014 (US Department of Health and Human Services, Centers for Disease Control and Prevention, National Center for Health Statistics, Washington, 2015).

3. Han, J. C., Lawlor, D. A. \& Kimm, S. Y. Childhood obesity. Lancet 375, 1737-1748 (2010).

4. Campion, J., Milagro, F. I. \& Martinez, J. A. Individuality and epigenetics in obesity. Obes. Rev. 10, 383-392 (2009). 
5. Isong I. A. et al. Racial and ethnic disparities in early childhood obesity. Pediatrics 141, https://doi.org/10.1542/peds.2017-0865 (2018).

6. Engeland, A. et al. Obesity in adolescence and adulthood and the risk of adult mortality. Epidemiology 15, 79-85 (2004).

7. Guo, S. S. \& Chumlea, W. C. Tracking of body mass index in children in relation to overweight in adulthood. Am. J. Clin. Nutr. 70, 145S-148S (1999).

8. Cartagena, D. C. et al. Factors contributing to infant overfeeding with hispanic mothers. J. Obstet. Gynecol. Neonatal Nurs. 43, 139-159 (2014).

9. Suchindran, $C$. et al. Association of adolescent obesity with risk of severe obesity in adulthood. JAMA 304, 2042-2047 (2010).

10. Smego, A. et al. High body mass index in infancy may predict severe obesity in early childhood. J. Pediatr. 183, 87 (2017). 93.e1.

11. Karaolis-Danckert, N. et al. Rapid growth among term children whose birth weight was appropriate for gestational age has a longer lasting effect on body fat percentage than on body mass index. Am. J. Clin. Nutr. 84, 1449-1455 (2006).

12. Baird, J. et al. Being big or growing fast: systematic review of size and growth in infancy and later obesity. BMJ 331, 929 (2005)

13. Demerath, E. W. et al. Rapid postnatal weight gain and visceral adiposity in adulthood: the Fels longitudinal study. Obesity (Silver Spring). 17, 2060-2066 (2009).

14. Young, B. E., Johnson, S. L. \& Krebs, N. F. Biological determinants linking infant weight gain and child obesity: current knowledge and future directions. Adv. Nutr. 3, 675-686 (2012).

15. Van Dijk, S. J. et al. Epigenetics and human obesity. Int. J. Obes. (Lond.). 39, 85-97 (2015).

16. Hazrati, S. et al. Clinical, social, and genetic factors associated with obesity at 12 months of age. J. Pediatr. 196, 175 (2018). 181.e7.

17. Hazrati, S. et al. Investigating the accuracy of parentally reported weights and lengths at 12 months of age as compared to measured weights and lengths in a longitudinal childhood genome study. BMJ Open. 6, e011653 (2016). 2016-011653.

18. Cohen, S., Kamarck, T. \& Mermelstein R. Perceived stress scale. Measuring stress: a guide for health and social scientists. 235-283 (Oxford University Press, Oxford, 1994).

19. Arthur, R. et al. AKT: ancestry and kinship toolkit. Bioinformatics 33, 142-144 (2017).

20. Auton, A. et al. A global reference for human genetic variation. Nature $\mathbf{5 2 6}, \mathbf{6 8}-74$ (2015).

21. Rasmussen, K. M., Catalano, P. M. \& Yaktine, A. L. New guidelines for weight gain during pregnancy: what obstetrician/gynecologists should know. Curr. Opin. Obstet. Gynecol. 21, 521-526 (2009).

22. World Health Organization. WHO Child Growth Standards: Methods and Development http://www.who.int/childgrowth/standards/technical_report/en/ (2006).

23. Rubin, L. H. Methods for handling missing data in the behavioral neurosciences: don't throw the baby rat out with the bath water. J. Undergrad. Neurosci. Educ. 5, A71-A77 (2007).

24. Pourhoseingholi, M. A., Baghestani, R. A. \& Vahedi, M. How to control confounding effects by statistical analysis. Gastroenterol. Hepatol. Bed Bench. 5, 79-83 (2012).
25. Steinley, D. K-means clustering: a half-century synthesis. Br. J. Math. Stat. Psychol. 59, 1-34 (2006).

26. Oken, E. et al. Maternal gestational weight gain and offspring weight in adolescence. Obstet. Gynecol. 112, 999-1006 (2008).

27. Chu, S. Y. et al. Gestational weight gain by body mass index among US women delivering live births, 2004-2005: fueling future obesity. Am. J. Obstet. Gynecol. 200, 271 (2009). e1,271.e7.

28. Wang, L. et al. Ethnic differences in risk factors for obesity among adults in California, the United States. J. Obes. 2017, 2427483 (2017).

29. Taylor, P. D. \& Poston, L. Developmental programming of obesity in mammals. Exp. Physiol. 92, 287-298 (2007).

30. Oelsner, K. T. et al. Maternal BMI as a predictor of methylation of obesity-related genes in saliva samples from preschool-age hispanic children at-risk for obesity. BMC Genom. 18, 57 (2017). 016-3473-9.

31. Linabery, A. M. et al. Stronger influence of maternal than paternal obesity on infant and early childhood body mass index: the Fels Longitudinal Study. Pediatr. Obes. 8, 159-169 (2013).

32. Li, A. et al. Parental and child genetic contributions to obesity traits in early life based on 83 loci validated in adults: the FAMILY study. Pediatr Obes. https://doi. org/10.1111/ijpo.12205, 133-140 (2018).

33. Taveras, E. M. et al. Racial/ethnic differences in early-life risk factors for childhood obesity. Pediatrics 125, 686-695 (2010).

34. Woo Baidal, J. A. et al. Reducing hispanic children's obesity risk factors in the first 1000 days of life: a qualitative analysis. J. Obes. 2015, 945918 (2015).

35. Heyman, M. B. \& Abrams, S. A. Section on Gastroenterology, Hepatology, and Nutrition, Committee on Nutrition. Fruit juice in infants, children, and adolescents: current recommendations. Pediatrics 139, https://doi.org/10.1542/ peds.2017-0967 (2017).

36. Trotter, L. J., Bowen, D. J. \& Beresford, S. A. Testing for racial/ethnic differences in the association between childhood socioeconomic position and adult adiposity. Am. J. Public Health 100, 1088-1094 (2010).

37. Jimenez-Cruz, A. et al. Low income, Mexican mothers' perception of their infants' weight status and beliefs about their foods and physical activity. Child Psychiatry Hum. Dev. 41, 490-500 (2010).

38. Boyington, J. A. \& Johnson, A. A. Maternal perception of body size as a determinant of infant adiposity in an African-American community. J. Natl. Med. Assoc. 96, 351-362 (2004).

39. Rich, S. S. et al. Perceptions of health status and play activities in parents of overweight hispanic toddlers and preschoolers. Fam. Community Health $\mathbf{2 8}$ 130-141 (2005).

40. Zilanawala, A. et al. Race/ethnic disparities in early childhood BMI, obesity and overweight in the United Kingdom and United States. Int J. Obes. (Lond.) 39, 520-529 (2015).

41. Kimbro, R. T., Brooks-Gunn, J. \& McLanahan, S. Racial and ethnic differentials in overweight and obesity among 3-year-old children. Am. J. Public Health 97, 298-305 (2007). 